\title{
POSTERIOR RIB RESECTION IN SCOLIOSIS
}

\author{
A Preliminary Report \\ Harry Piggott, Birmingham, England \\ From the Royal Orthopaedic Hospital, Birmingham, and the \\ Department of Surgery, University of Birmingham
} \begin{abstract}
scoliosis this opinion could not be considered conclusive. the normal spine, bending it towards the side of operation. If the same forces were invoked in the already deformed spine it might be possible, by operating on the ribs or transverse processes in the concavity, to introduce a corrective force. Therapeutic value can only be assessed in the scoliotic patient, and before attempting this it seemed desirable to investigate further the mechanism whereby this type of operation deforms the spine; and in particular to find out whether soft-tissue operation alone would suffice or if removal of bone was essential, and if so, which part of the bone.
\end{abstract}

Bisgard (1934) reported lateral spinal curvature as a common occurrence after thoracoplasty: he showed that the worse deformities occurred when rib resection was carried far posteriorly and the transverse process was removed. In 1961 Langenskiöld and Michelsson reported that scoliosis could regularly be produced in young animals by unilateral excision of the heads and necks of six adjacent ribs, and somewhat less consistently by other operations in the same area, notably resection of short segments of rib shafts immediately lateral to the transverse processes. Later (Langenskiöld and Michelsson 1962) they expressed the view that these operations initiated deformity by interfering with the function of the superior costotransverse ligaments, but as excision of these ligaments alone was only rarely followed by

The mechanism may be debatable, but it is apparent from these reports that surgical intervention in the costo-transverse region can in some way introduce forces which deform

\section{EXPERIMENTAL SCOLIOSIS}

Method-Rabbits aged seven to twenty-one days were divided into three groups. In all, the posterior parts of the left sixth to eleventh ribs inclusive were exposed: in the first group the head and neck of each rib was excised; in the second group short segments of rib shaft immediately lateral to the transverse process were resected; and in the third group the soft-tissue procedure was performed, including mobilisation of the heads and necks, but no bone was resected, the ribs being carefully replaced in position.

All animals were killed after attaining skeletal maturity and radiographs of the whole spine and thorax were made (Fig. 1). Scoliotic curves when present were measured by the method of Cobb (1948). After radiography the spine and thoracic cage were dissected free

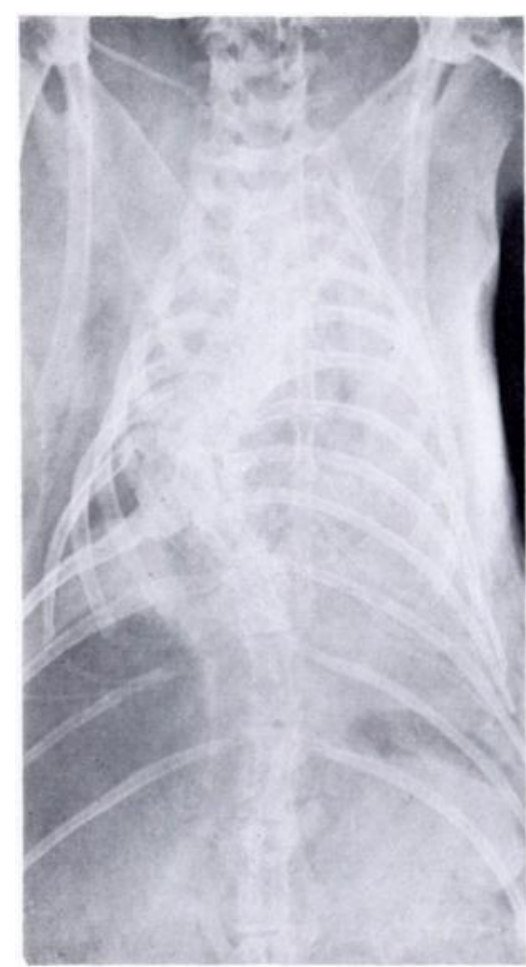

FIG. 1

Moderately severe scoliosis in a rabbit after resection of the heads and necks of the left sixth to eleventh ribs at the age of 7 days. 

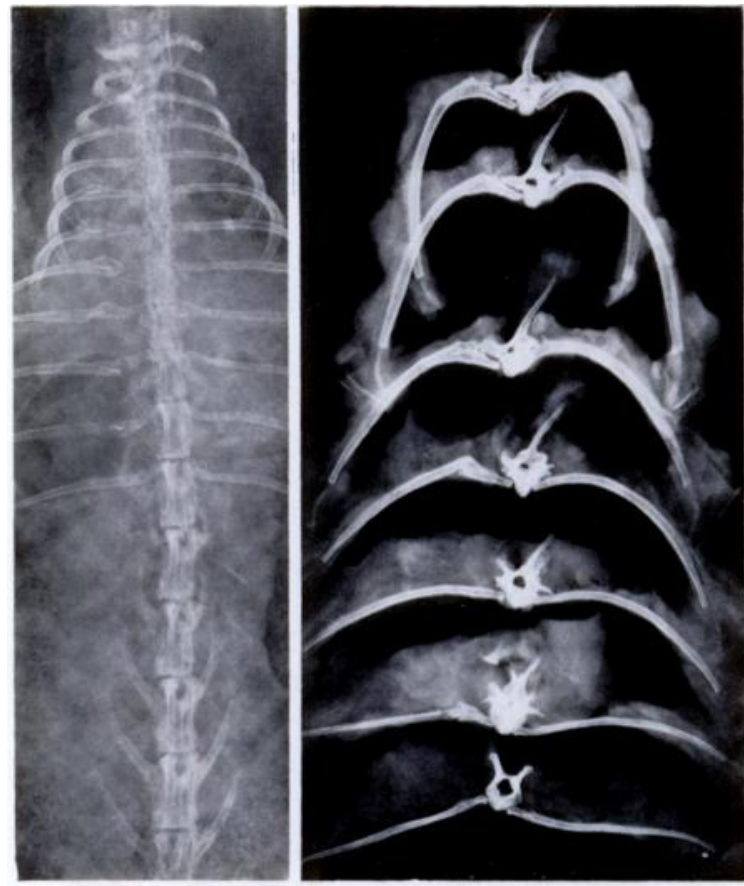

Fig. 2
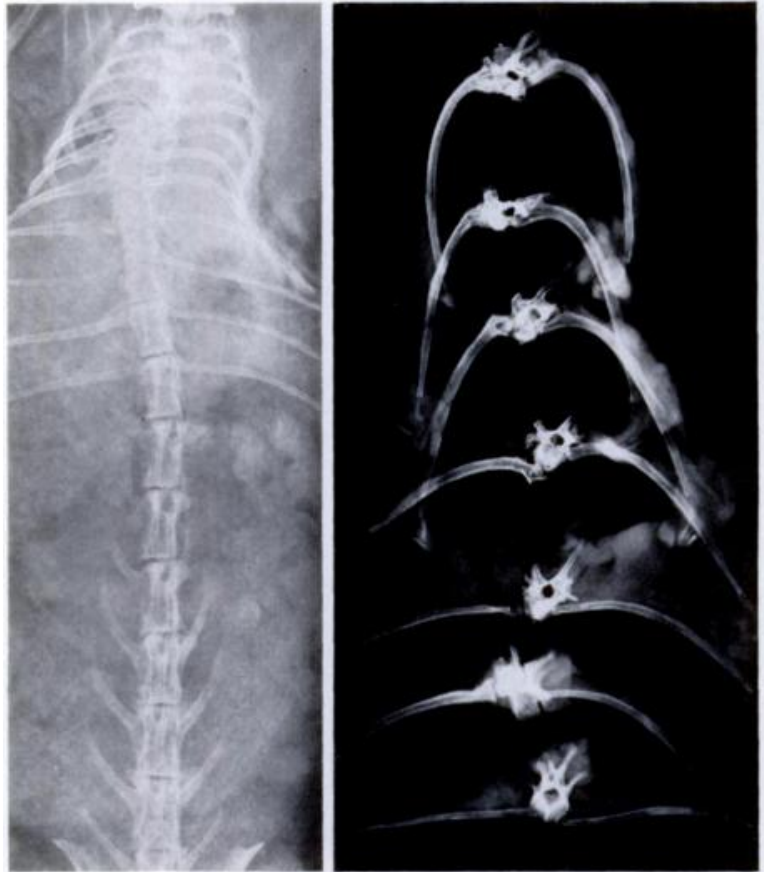

Fig. 3

Figure 2 -Result of resection of short segments of the left sixth to eleventh ribs just lateral to the transverse processes. There has been excellent reconstitution of the ribs and no scoliosis: markers implanted at the distal cut ends confirm that normal growth of the posterior parts of the ribs has continued. Figure 3-Result of resection of the heads and necks of the left sixth to eleventh ribs. Failure of regeneration and growth, with scoliosis. 
and split up into segmental units, each consisting of a vertebra with its attached pair of ribs, and in the upper segments the corresponding section of sternum, and further radiographs were made of the individual skeletal segments (Figs. 2 and 3).

RESULTS

The results are shown in Table I. It will be seen that with few exceptions significant scoliosis occurred only after head and neck resection, when it was almost constant, though a few mild curves developed in the other two groups. This fact, that removal of the head and

TABLE I

Spinal Curvatures Following Operation on Ribs in Young Rabitts

\begin{tabular}{|c|c|c|c|}
\hline Operation & $\begin{array}{l}\text { Number of } \\
\text { animals }\end{array}$ & Degrees of curvature produced & Average \\
\hline $\begin{array}{l}\text { Rib head and neck } \\
\text { resection. }\end{array}$ & 19 & 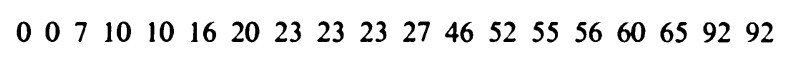 & 35 \\
\hline Rib segment resection & 14 & $\begin{array}{llllllllllllll}0 & 0 & 0 & 0 & 0 & 0 & 0 & 5 & 5 & 12 & 12 & 16 & 18 & 49\end{array}$ & $8 \cdot 5$ \\
\hline Rib stripping . & 10 & $\begin{array}{llllllllll}0 & 0 & 0 & 0 & 4 & 7 & 8 & 9 & 15 & 22\end{array}$ & 5.9 \\
\hline
\end{tabular}

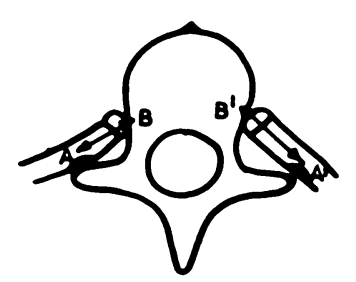

FIG. 4

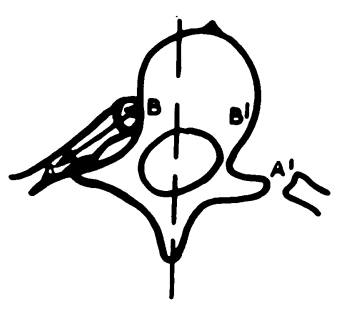

Fig. 6

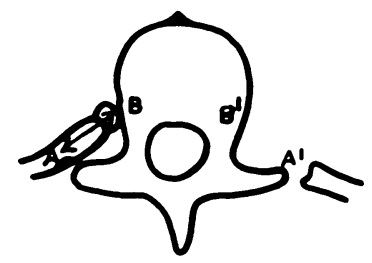

FIG. 5

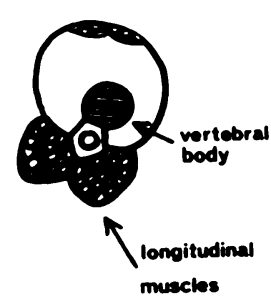

FIG. 7

Figure 4-A normal thoracic vertebra with its attached ribs. Figure 5-One rib head and neck has been removed, giving rise to asymmetrical mechanical and growth thrust. Figure 6 -Suggested rotational deformity resulting from Figure 5. Figure 7-Fixed rotation causes asymmetrical longitudinal compression of the vertebral growth plates, so that lateral wedging and curvature may follow.

neck was the only manoeuvre consistently producing scoliosis, suggests that unilateral lack of mechanical support of the spine by the ribs, or arrest of growth at the posterior end of the ribs, or both factors combined, may contribute to the deformity. To check that retardation of rib growth occurred in the head and neck resection group but not in the others, metal markers had been implanted at the cut ends of the ribs at the time of resection. Figures 2 and 3 show typical examples which confirm the usual findings of cessation of posterior growth after head and neck resection, but remarkable reconstitution of bone after segmental resection.

VOL. 53 B, NO. 4, NOVEMBER 1971 
DISCUSSION

It is suggested that unilateral arrest of rib growth and lack of support of the spine are concerned in initiating these experimental curvatures, and the following mechanical considerations show that lateral curvature and rotation may both be involved de novo. As the operation creates a gap in the bony skeleton along one side of the spine, mechanical pressure from the contralateral intact ribs may push the mobile column over into the gap, and subsequently asymmetrical rib growth may fix and increase the deformity. Arkin (1950) has shown that forced lateral curvature of the spine will of itself cause rotation, but another mechanism is probably at work in the experimental deformities considered here whereby the reverse occurs, an initial rotation giving rise to lateral curvature. Figure 4 illustrates how the vertebral body is gripped symmetrically by the ribs, and it must be remembered that in the growing animal it is not only muscular and mechanical stresses that are transmitted through the ribs to the vertebrae, but also the more gradual, though perhaps more important, moulding forces of bone growth. If growth is arrested at the rib heads on one side, it seems inevitable that some degree of asymmetry of vertebral growth will follow, and Figures 5 and 6 suggest the form this would be most likely to take: that is, if arrest of growth along the line of the rib head and neck on one side is followed by retardation of growth of the vertebra in the same line while contralateral growth continues at the normal rate, then the vertebra must grow into the position of rotatory deformity towards the side of growth arrest.

As Figure 7 illustrates, once rotation has appeared, the growth plates of the vertebral bodies are situated asymmetrically in relation to longitudinal muscle action (and gravity in an orthograde animal) in such a way that they are subject to relative compression on the side away from which they are rotated; retardation of longitudinal growth will therefore occur on this side, giving rise to lateral curvature.

Thus, removal of the heads and necks of the ribs can set in motion a train of mechanical and growth forces which will cause lateral curvature and rotation of the spine towards the side of operation; but this does not account for the occasional development of curves as a result of soft-tissue procedures or less radical bone resection in the same area, slight in the present study, but severe in the hands of some workers.

The findings of MacEwen (1968) and Wyke (1970) may explain these curves. MacEwen showed that unilateral section of posterior nerve roots in young animals could produce structural scoliosis, and Wyke found that the area around the costo-vertebral and costotransverse joints was very rich in proprioceptive nerve endings. It is therefore possible that operations in this area may, by cutting off proprioceptive impulses, reflexly produce asymmetrical muscle activity with resulting deformity. This mechanism of course may well be active in the bone resection operations in addition to the mechanical and growth derangements already discussed.

The two rabbits in the head and neck resection group whose spines did not develop significant curvature are also of considerable interest in connection with etiological mechanisms. They were exceptional in showing remarkably accurate regeneration of the posterior ends of the ribs; so much so that at necropsy it was difficult to believe that the rib heads and necks had been excised. One was forced to conclude that perhaps small peripheral fragments of the epiphysis escaped excision, and in these very young animals had been able to proliferate and remodel a growing bone end. Whatever the explanation of this reconstitution of the heads and necks, the finding that it was associated with an absence of spinal deformity lends support to the view that suppression of this part of the bone is an important factor in the pathogenesis of these deformities.

Relevance to human scoliosis-The object of this work was to investigate a possible method of treatment, and any relevance these observations may have to the etiology of human scoliosis is purely speculative. So far as idiopathic and congenital curves are concerned, it seems unlikely that the mechanisms discussed in this paper are involved, but they may play a part 
in the pathogenesis of paralytic curves. James (1956) showed that in curves following poliomyelitis intercostal muscle paresis was always more severe on the convex side; this muscle imbalance, possibly combined with imbalance of the spinal musculature, would directly produce spinal curvature.

But other mechanisms may well contribute, and by analogy with the retarded limb growth following paralysis we might expect retardation of rib growth on the weaker (convex) side. Further, the vertical position of these ribs in curves following poliomyelitis must diminish their lateral thrust on the spinal column (Fig. 8), so that both retarded growth and diminished

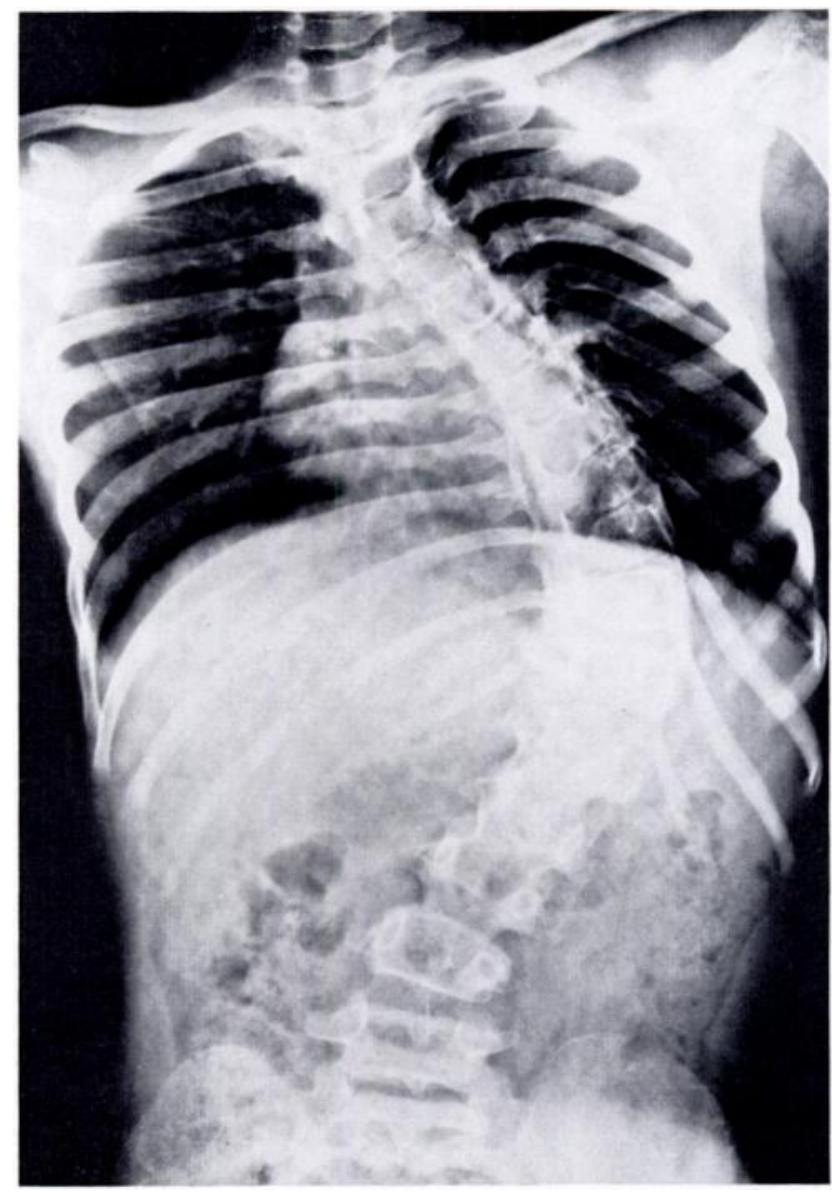

FIG. 8

A curve caused by poliomyelitis. Note the greatly increased vertical inclination of the ribs on the convexity, especially at the apex of the curve.

mechanical support may be etiological factors in these curves in addition to the more obvious muscle imbalance. Similarly, loss of proprioceptive impulses may have little relevance to idiopathic and congenital curves, but as MacEwen (1968) has pointed out it could be highly relevant to some neurogenic curves, for instance in syringomyelia.

In spite of the uncertainty about pathogenesis of these experimental deformities and whether similar processes are etiological in some human curves, the fact remains that resection of the posterior ends of several adjacent ribs in growing animals introduces a system of forces capable of rotating and bending the spine towards the side of operation. It remains to be seen whether or not the same operation done on the concave side of the already deformed 
spine will, by introducing the same forces, exert a corrective influence. One cannot conclude from the experimental work that this therapeutic result will follow, because the rabbits, unlike scoliotic children, had normal anatomy, function and growth potential on the unoperated side. Still less can one anticipate a uniform result, since scoliosis is not a uniform disease, nor a uniform mechanical problem. Normal spinal shape depends on many factors, of which bone growth and neuromuscular action are two of the more obvious, and there is a complex balance of forces preserving normal symmetrical spinal development which may be disturbed in many ways, with resulting deformity. Rib head and neck resection could at best be regarded as another force dropped into the scales, of unknown quantity, but at least on the right side, and its therapeutic value, if any, can only be assessed by studying its effect in scoliotic children.

\section{THERAPEUTIC TRIAL}

Selection of patients-Because the procedure was untried it seemed desirable to assess the results of this operation first in cases where it was expected that the outcome of orthodox treatment might be unsatisfactory: these have been found mainly in the infantile and juvenile idiopathic groups. Perseverance with the Milwaukee brace until these younger children are old enough for fusion will control most curves, but a few cannot tolerate bracing, some progress in spite of it, and even those who have an acceptable result have paid the heavy price of a complete childhood restricted by a brace. Another reason for selecting the younger patients is that with many growing years ahead, a procedure which modifies growth should have more chance of success. For these reasons the operation was limited to children whose curves were known to be progressive, usually rapidly so, often in spite of bracing, and with few exceptions in the younger patients.

Thus only a small proportion of scoliotic children treated have been considered suitable for this operation, which has been performed in twenty-seven cases, the average age being six years and five months (range two years and seven months to fourteen years). Fifteen were boys and twelve girls, as would be expected in a group of predominantly early onset curves. All curves were thought before operation to be idiopathic, but in one case (Case 11 in Table II) neurofibromatous tissue was found along several intercostal nerves, and another (Case 16 in Table II) showed extensive fibrosis of the erector spinae on the concave side, fibrous strands fanning out from the tip of the transverse process at the apex of the curve. In this case there was no clue to the etiology of the fibrosis, which appeared to be the direct cause of the curve.

\section{TECHNIQUE OF OPERATION}

The object is to excise the heads and necks of the ribs in the concavity of the curve; this has usually been the sixth to eleventh inclusive, but on occasion has moved up or down one segment. A posterior incision starts in the midline two spinous processes cephalic to the upper level of resection, curves laterally on the concave side, then back to the midline at the lower limit of the curvature. The superficial muscles are divided in the line of the incision and reflected medially. The lateral margin of the erector spinae is identified and its attachments to the ribs are divided to permit mobilisation of the muscle mass medially as far as the transverse processes. This exposes the ribs, which are freed subperiosteally one to two inches lateral to the transverse processes, and are divided at this point. Subperiosteal dissection then proceeds medially as far as the cartilaginous rib head; on the superior, posterior and inferior aspects of the neck sharp dissection is required to free the many ligamentous attachments, but elsewhere the periosteum strips easily. Great care is required throughout to avoid puncturing the pleura. If the freed posterior segment of rib is now twisted around its longitudinal axis, the metaphysis separates readily from the cartilaginous head, which at this stage remains in place. The intercostal nerves are then identified immediately inferior to the ribs, and exposed as far as the intervertebral foramina; the 


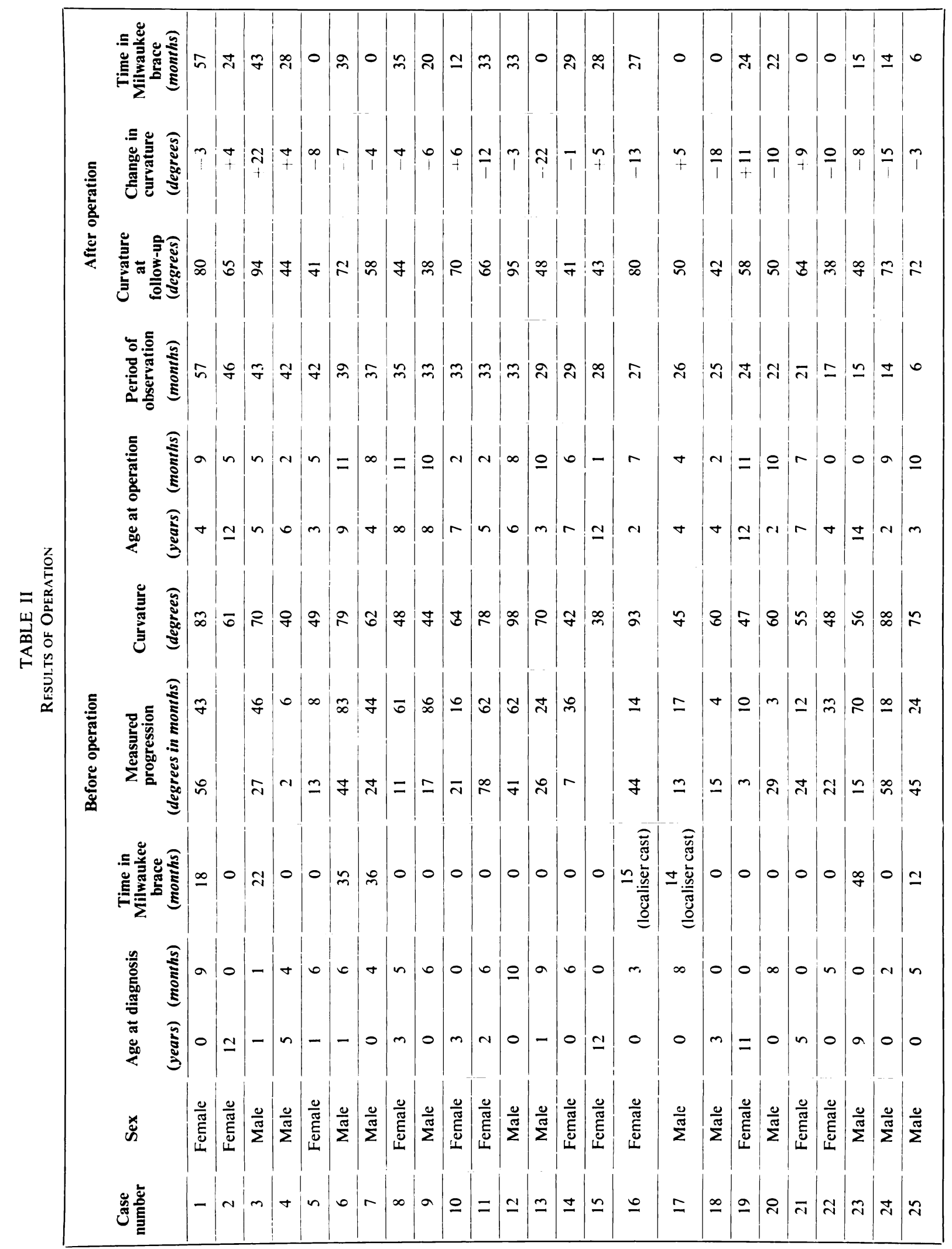

VOL. 53 B, NO. 4, NOVEMBer 1971 
intercostal soft tissues can then be safely detached from the transverse processes and mobilised forward to permit good exposure of the rib heads. The ligaments of the costo-vertebral joints, which attach the rib heads to the vertebral bodies, are then divided and the rib heads removed.

The rotation of the deformed spine makes access to the apical rib heads sometimes difficult, and a transpleural approach would be easier, but it has been felt desirable to avoid opening of the pleura if possible, for if the whole procedure remains extrapleural there is remarkably little disturbance to the patient and the time in hospital is short.

Complications - In one severely deformed child the operation was abandoned in the early stages because of rapid unexplained deterioration in respiratory state; this patient recovered uneventfully, but is excluded from the follow-up. In three cases small pleural tears were made and intercostal drainage was needed for forty-eight hours; all recovered uneventfully. There have been no other complications and no deaths.

RESULTS

The operation has been performed twenty-seven times. One child left the country shortly after operation and is lost to follow-up. The boy on whom the operation was abandoned is also excluded, leaving twenty-five cases for study, details of which are given in Table II. The length of observation is from six to fifty-seven months, with an average of twenty-nine months. The average curve before operation was 64 degrees (range 38 to 98 degrees), and at the latest follow-up the average was 58.5 degrees (range 38 to 95 degrees).

\section{DISCUSSION}

It is apparent from these averages and from Table II that whereas all curves were progressive before operation there has been but little progression after it, and several curves have shown significant retrogression. It thus appears that operation has had overall a favourable influence, but many variables are involved and it must be stressed that in this relatively early attempt at assessment, in spite of apparently impressive improvement in some cases, caution must be observed in interpretation. As we are treating children with a potentially crippling deformity it has not been justifiable to attempt a controlled trial of treatment nor to adopt an untried operation as a standard procedure, and at all stages in every case an attempt has been made to do what seemed best for that particular child at that time. Thus, these twentyseven cases represent only a small proportion of the total number of patients seen and treated; it is not possible to find similar cases untreated for comparison, and many of the twenty-seven have had subsequent additional therapy, either bracing or posterior fusion (in Table II, for patients who have had further operation, follow-up ends at that time). With the knowledge that scoliosis progresses at different rates at different ages, that curves may for relatively long intervals remain static, and that the effect of the Milwaukee brace depends on enthusiasm and attention to detail by maker, family and surgeon, it is possible on a rigid analysis to attribute most of the improvements to causes other than the operation. However, in patients who had progressive curves before operation, showed retrogression after operation, and at no stage had any other form of treatment, the conclusion cannot be escaped that the operation has directly produced an improvement in the deformity.

There have been five cases fulfilling these criteria and it is interesting that all had operation early, before the age of five years; the best of these progressed 26 degrees in the twenty-four months preceding operation, and subsequently retrogressed 22 degrees in twenty-nine months after operation. Even here a cautionary note must be sounded; the longest follow-up in this very small group is forty-two months, and there have been a few cases in older children with longer follow-up in whom dramatic continuous initial improvement has been followed by subsequent relapse. 
It has also been disappointing that no apparent improvement in rotation has been produced. This is difficult to express numerically but as judged from appearance and from radiographs there has been no case in which rotation has been less after operation than it was before.

\section{SUMMARY}

1. The production of scoliosis in young animals by resection of the posterior ends of the ribs is described and the etiological mechanisms discussed. It is suggested that retardation of posterior rib growth, removal of mechanical support from one side of the spine, and disturbance of proprioceptive impulses are the factors which initiate the deformity.

2. Some or all of these mechanisms may contribute to the production of certain human curves, for instance impaired rib growth and support in post-poliomyelitic deformities, proprioceptive and reflex disturbances in syringomyelia.

3. Therapeutically resection of the heads and necks of the ribs on the concave side of thoracic idiopathic curves is followed by improvement in some cases. Exactly how much improvement, in what proportion of curves, and for how long it will be maintained cannot be defined at present, but therapeutically worthwhile effects have been obtained, especially in children under five years old; even if this improvement is followed by later progression, several years of freedom from bracing may have been achieved, and the operation has not in any way interfered with subsequent correction and posterior fusion if this has seemed necessary. It is thought that this operation may have a small but useful rôle in the early management of idiopathic scoliosis, especially in a young child who is not responding well to bracing.

It is a pleasure to acknowledge my indebtedness to Professor A. L. d'Abreu in whose department the experimental work was carried out, to the Birmingham Regional Hospital Board who generously financed it, and to the Photographic Department of the Children's Hospital, Birmingham.

\section{REFERENCES}

ARkin, A. M. (1950): The Mechanism of Rotation in Combination with Lateral Deviation in the Normal Spine. Journal of Bone and Joint Surgery, 32-A, 180.

BisGaRD, J. D. (1934): Thoracogenic Scoliosis: Influence of Thoracic Disease and Thoracic Operations on the Spine. Archives of Surgery, 29, 417.

Совв, J. R. (1948): Outline for the Study of Scoliosis. In Instructional Course Lectures, The American Academy of Orthopaedic Surgeons. Vol. 5, pp 261-275. Ann Arbor: J. W. Edwards.

James, J. I. P. (1956): Paralytic Scoliosis. Journal of Bone and Joint Surgery, 38-B, 660.

Langenskiöld, A., and Michelsson, J.-E. (1961): Experimental Progressive Scoliosis in the Rabbit. Journal of Bone and Joint Surgery, 43-B, 116.

Langenskiöld, A., and Michelsson, J.-E. (1962): The Pathogenesis of Experimental Progressive Scoliosis. Acta Orthopaedica Scandinavica, Supplementum 59.

MacEwen, G. D. (1968): Experimental Scoliosis. In Proceedings of a Second Symposium on Scoliosis: Causation, p. 18. Edited by P. A. Zorab. London and Edinburgh: E. \& S. Livingstone Ltd.

WYKE, B. (1970): Personal communication. 